\title{
PAPER \\ Decentralized Probabilistic Frequency-Block Activation Control Method of Base Stations for Inter-cell Interference Coordination and Traffic Load Balancing*
}

\author{
Fumiya ISHIKAWA $^{\dagger}$, Student Member, Keiki SHIMADA ${ }^{\dagger}$, Nonmember, Yoshihisa KISHIYAMA ${ }^{\dagger \dagger}$, \\ and Kenichi HIGUCHI ${ }^{\dagger a)}$, Senior Members
}

\begin{abstract}
SUMMARY In this paper, we propose a decentralized probabilistic frequency-block activation control method for the cellular downlink. The aim of the proposed method is to increase the downlink system throughput within the system coverage by adaptively controlling the individual activation of each frequency block at all base stations (BSs) to achieve inter-cell interference coordination (ICIC) and traffic load balancing. The proposed method does not rely on complicated inter-BS cooperation. It uses only the inter-BS information exchange regarding the observed system throughput levels with the neighboring BSs. Based on the shared temporal system throughput information, each BS independently controls online the activation of their respective frequency blocks in a probabilistic manner, which autonomously achieves ICIC and load balancing among BSs. Simulation results show that the proposed method achieves greater system throughput and a faster convergence rate than the conventional online probabilistic activation/deactivation control method. We also show that the proposed method successfully tracks dynamic changes in the user distribution generated due to mobility.

key words: heterogeneous networks, probabilistic activation control, transmission power control
\end{abstract}

\section{Introduction}

In the 5th generation mobile communication system [1]-[3], in order to actualize high capacity at low cost, heterogeneous networks [4], [5] in which low-transmission-power pico-base stations (BSs) are overlaid onto the coverage area of a high-transmission-power macro-BS are considered to be an essential component. However, the use of an excessively large number of pico-BSs may cause severe inter-cell interference. Therefore, appropriate activation of each picoBS depending on the user or traffic load distribution within the system coverage is important in order to achieve full system performance.

In [6], heuristic BS activation/deactivation methods were proposed. These methods turn off lightly-loaded BSs.

Manuscript received October 28, 2019.

Manuscript revised February 25, 2020.

Manuscript publicized April 2, 2020.

${ }^{\dagger}$ The authors are with the Department of Electrical Engineering, Graduate School of Science and Technology, Tokyo University of Science, Noda-shi, 278-8510 Japan.

${ }^{\dagger}$ The author is with NTT DOCOMO, INC., Yokosuka-shi, 2398536 Japan.

*The material in this paper was presented in part at the IEEE 90th Vehicular Technology Conference, Honolulu, U.S.A., September 2019.

a)E-mail: higuchik@rs.tus.ac.jp

DOI: $10.1587 /$ transcom.2019EBT0006
However, in reality, the users connected to the deactivated BSs should be handed over to other neighboring BSs in order to guarantee the quality of service $(\mathrm{QoS})$ of these users. Thus, the activation/deactivation control of a certain BS (cell) affects the performance of the neighboring cells. These BS activation/deactivation methods are insufficient since the above issue is not fully taken into account. In [7] and [8], probabilistic BS activation/deactivation control methods were proposed. These methods effectively achieve optimal BS activation/deactivation control taking into account the coupled performance among cells caused by the activation/deactivation control of individual BSs. However, they require the prior knowledge of probability distributions of the user locations or the traffic load of the entire system coverage, which is in reality very difficult to achieve.

Members of our research group have recently reported a method for the online probabilistic activation/deactivation control of pico-BSs (picocells) in heterogeneous networks [9], [10]. We focus on the maximization of the system throughput. The reported method does not rely on $a$ priori knowledge of probability distributions of user locations or the traffic load of the entire system coverage. Therefore, complicated inter-BS cooperation and measurement at the user terminals are not necessary. This also enables the online activation/deactivation control of pico-BSs that track the changes in user/traffic distribution within the system coverage. The reported method requires only a single metric to be exchanged among BSs, which is the system throughput measured at each cell. The reported method adaptively controls the activation probability of each pico-BS individually using an iterative algorithm, depending on the time variation in the system throughput and the temporal activation/deactivation states of each BS. In the following, the method in [10] is referred to as the conventional method.

In order to improve the convergence rate and stability after convergence in the iterative algorithm of the conventional method, the range in inter-BS information exchange regarding the observed system throughput is investigated in [11]. Appropriately limiting the range in inter-BS information exchange regarding the local system throughput is beneficial in reducing undesirable control when updating the activation probability of a particular BS based on the system throughput observed far away from that BS, which is effectively not related to the activation/deactivation state of 
that BS. Hereafter, this approach is referred to as the limited range in inter-BS information exchange (LRIE). Furthermore, an adaptive step size control (ASSC) that updates the activation probability was investigated in [12]. The ASSC brings about improvement in the convergence rate of the iterative algorithm and increases the system throughput after convergence.

In this paper, we propose a novel frequency blockdependent online probabilistic activation control for BSs in heterogeneous networks. In the proposed method, the overall system bandwidth is divided into multiple frequency blocks. The proposed method independently performs activation control on a per frequency block basis at each BS based on the temporal variation of the observed system throughput similar to the conventional method in [10]. By performing the frequency block-dependent activation control, we can increase the granularity of the activation/deactivation control and achieve an inter-cell interference coordination (ICIC) effect [13] in addition to the overall reduction in inter-cell interference. The concept of using multiple frequency blocks has been used in several ICIC methods such as fractional frequency reuse [14]. The proposed method in this paper is a practical method that activates/deactivates multiple frequency blocks of all BSs in a decentralized way by only relying on the inter-BS systemthroughput information exchange. The proposed method achieves online activation control that tracks the dynamic fluctuation in the user position over time and achieves ICIC and traffic load balancing. This is the difference that sets the proposed method apart from existing work using multiple frequency blocks.

In the proposed method, the higher granularity in the activation/deactivation control may result in a longer convergence time. To address this problem, we propose a method in which the number of activated frequency blocks is controlled in a probabilistic manner in addition to the control in activation probability of each frequency block. Furthermore, the concepts behind the LRIE and ASSC are integrated into the proposed method. We note that our initial investigations on the contents of this paper are presented in [15] and [16]. This paper is an enhanced version of [16] with more detailed evaluation and discussions. Computer simulation results, in which the mobility of the user terminals is taken into account, show the effectiveness of the proposed method.

The remainder of the paper is organized as follows. Section 2 describes the system model. Then, Sect. 3 presents the conventional method including the LRIE and ASSC. Section 4 presents the proposed method. Section 5 presents numerical results on computer simulations. Finally, Sect. 6 concludes the paper.

\section{System Model}

The set of all BSs in the system is denoted as $\mathcal{N}_{\mathrm{BS}}$. The set of macro-BSs in the system is denoted as $\mathcal{N}_{\text {macro }}$, while the set of pico-BSs in the system is denoted as $\mathcal{N}_{\text {pico }}\left(\mathcal{N}_{\text {pico }}=\right.$
$\left.\mathcal{N}_{\mathrm{BS}} \backslash \mathcal{N}_{\text {macro }}\right)$. In Sect. 5, we evaluate two frequency reuse schemes between macro- and pico-BSs. The first one assumes that the same frequency is used at both macro- and pico-BSs and the second one assumes that different frequencies are applied to macro- and pico-BSs. In both cases, we assume the same overall bandwidth per BS, $w_{\text {tot }}$, for simplicity. The overall system frequency bandwidth is divided into multiple frequency blocks, and the set of frequency blocks in the system is denoted as $\mathcal{F}$. The bandwidths of all frequency blocks are assumed to be equally set to $w_{\text {tot }} /|\mathcal{F}|$. The transmission power densities of the macro- and picoBSs are denoted as $p_{\text {macro }}$ and $p_{\text {pico }}$, respectively. The transmission power density of $\mathrm{BS} n$ is denoted as $p_{n}$. When $n \in \mathcal{N}_{\text {macro }}, p_{n}=p_{\text {macro }}$, while when $n \in \mathcal{N}_{\text {pico }}, p_{n}=p_{\text {pico }}$. The set of users in the system is denoted as $\mathcal{K}$. Hereafter, the unit of discrete time, $t$, is set as the transmission activation/deactivation control cycle. The user association is assumed to be updated at every $t$ based on the cell range expansion (CRE) method [17] depending on the activation states of all BSs at all frequency blocks. The set of users served by BS $n$ at time $t$ is denoted as $\mathcal{K}_{n}[t]$. The BS index to which user $k$ is connected at time $t$ is denoted as $n_{k}[t]$. The received signal-to-interference-plus-noise ratio (SINR) of user $k$ in frequency block $f$ at time $t$ is denoted as $\gamma_{k, f}[t]$, which is dependent on the channel conditions between user $k$ and all (serving and interfering) BSs, activation states of all BSs, and frequency reuse strategy between macro- and pico-BSs.

Assuming that user $k$ is allocated a fraction of the frequency bandwidth, $w_{k, f}[t]$, of the respective frequency blocks $f$ from BS $n_{k}[t]$ at time $t$, the throughput of user $k$ at time $t, c_{k}[t]$, is represented as

$$
c_{k}[t]=\sum_{f \in \mathcal{F}} w_{k, f}[t] \log _{2}\left(1+\gamma_{k, f}[t]\right) .
$$

In this paper, we define the system throughput based on proportional fairness [18]; thus, the system throughput is defined by the geometric mean user throughput, which is equivalent to the log-sum of the user throughput. Proportional fairness is widely used in actual systems since it achieves a good tradeoff between the spectrum efficiency and fairness among users. The system throughput observed at each $\operatorname{BS~} n, C_{n}[t]$, is represented as

$$
C_{n}[t]=\left(\prod_{k \in \mathcal{K}_{n}[t]} c_{k}[t]\right)^{1 /\left|\mathcal{K}_{n}[t]\right|} .
$$

In this paper, we assume orthogonal multiple access, since orthogonal frequency division multiple access (OFDMA) is used in LTE and LTE-Advanced and is assumed as a baseline in the 5th generation mobile communication system. From [19], the optimum bandwidth allocation to user $k$ at each frequency block $f$ of BS $n_{k}[t], w_{k, f}[t]$, that maximizes $C_{n}[t]$ is represented as 


$$
w_{k, f}^{*}[t]=\max \left(0, \frac{1}{\mu_{f}}-\frac{\sum_{i \in \mathcal{F} \backslash\{f\}} w_{k, i}^{*}[t] \log _{2}\left(1+\gamma_{k, i}[t]\right)}{\log _{2}\left(1+\gamma_{k, f}[t]\right)}\right),
$$

where Lagrangian multiplier $\mu_{f}$ is determined to satisfy the following equation.

$$
\sum_{k \in \mathcal{K}_{n}[t]} w_{k, f}^{*}[t]=w_{\text {tot }} /|\mathcal{F}|
$$

\section{Conventional Method and LRIE/ASSC}

\subsection{Conventional Method}

The conventional method in [10] activates or deactivates all the frequency blocks simultaneously at each BS. In other words, $|\mathcal{F}|$ is set to one in the conventional method. The purpose of the activation/deactivation control in the conventional method is to reduce the inter-cell interference power by deactivating unnecessary BSs in order to increase the system throughput. Since the conventional method controls the activation/deactivation state iteratively and independently at each pico-BS, we will describe hereafter the control at a certain pico-BS $n$.

The system throughput within the system coverage observed at discrete time $t$ is denoted as $\bar{C}[t]$. We assume that $\bar{C}[t]$ is shared by all BSs in the system using the inter-BS information exchange via the backhaul. At time $t$, the activation probability of pico-BS $n$ is denoted as $q_{n}[t]$, whose control is the purpose of the conventional method. To simplify the description of the proposed method, we denote the activated and deactivated states as ' 1 ' and ' 0 ', respectively. The activation/deactivation state of pico-BS $n$ at time $t$ is represented by $s_{n}[t] \in\{1,0\}$. Based on the observation of the system throughput at time $t-1$ and $t$, the change in the system throughput from time $t-1$ to $t, \Delta \bar{C}[t]$, is calculated as

$$
\Delta \bar{C}[t]=\frac{\bar{C}[t]-\bar{C}[t-1]}{\bar{C}[t]} .
$$

In the conventional method, $q_{n}[t]$ is updated by the following equation based on $\Delta \bar{C}[t]$ and $s_{n}[t]$.

$$
q_{n}[t+1]=q_{n}[t]+\varepsilon \cdot \operatorname{sign}(\Delta \bar{C}[t]) \cdot\left(s_{n}[t]-s_{n}[t-1]\right) .
$$

Term $\varepsilon$ is a small positive constant that corresponds to the step size when updating $q_{n}[t]$. Term $q_{n}[t+1]$ is calculated using (6). However, when $q_{n}[t+1]$ reaches 1.0 or 0.0 , the activation/deactivation states of BS $n$ are fixed after $t+1$. To avoid this, the upper and lower limits of $q_{n}[t]$ are limited to $1-\rho$ and $\rho$, respectively. Term $\rho$ is a sufficiently small positive number.

Updating $q_{n}[t]$ based on (6) means that when the activation/deactivation states of pico-BS $n$ change from time $t-1$ to $t$ (thus, $s_{n}[t] \neq s_{n}[t-1]$ ) and system throughput $\bar{C}$ is increased from $t-1$ to $t$ (thus, $\Delta \bar{C}[t]>0$ ), $q_{n}[t]$ is updated so that state $s_{n}[t]$ is retained with high probability at time $t+1$. That is, if pico-BS $n$ is activated at time $t$ (thus, if $s_{n}[t]$ is 1$), q_{n}[t+1]$ is increased by $\varepsilon$ from $q_{n}[t]$. If picoBS $n$ is deactivated at time $t$ (thus, if $s_{n}[t]$ is 0 ), $q_{n}[t+1]$ is decreased by $\varepsilon$ from $q_{n}[t]$. When system throughput $\bar{C}$ is decreased from $t-1$ to $t$ (thus, $\Delta \bar{C}[t]<0$ ), the opposite control is performed on $q_{n}[t]$ so that state $s_{n}[t]$ tends to change with high probability at time $t+1$. When $s_{n}[t]=s_{n}[t-1]$, thus when the activation/deactivation states of pico-BS $n$ did not change from time $t-1$ to $t, q_{n}[t+1]$ remains as $q_{n}[t]$ since the value of $\Delta \bar{C}[t]$ is considered to be unrelated to the current activation/deactivation state of pico-BS $n$.

Then, the activation or deactivation of the respective pico-BSs at time $t+1$ is decided in a probabilistic manner using $q_{n}[t+1]$. The user association is re-performed among macro-BSs and the activated pico-BSs based on the determined activation/deactivation states of all the BSs. By repeating this process over time, activation/deactivation control of all BSs capable of increasing system throughput $\bar{C}$ for a given distribution of user terminals is achieved.

\subsection{LRIE}

The reason why the system throughput information of neighboring BSs is used at each pico-BS $n$ in updating $q_{n}[t]$ is to take into account the impact of the activation states of that BS on the performance of neighboring BSs since the inter-cell interference and traffic load balancing are dependent on it. However, an excessively wide range in inter-BS information exchange may increase the amount of traffic in the backhaul and the activation/deactivation control delay. Furthermore, in the conventional method, updating the activation probability of a particular BS based on the system throughput observed far away from that BS, which is effectively not related to the activation/deactivation state of that $\mathrm{BS}$, may result in undesirable control.

The LRIE appropriately limits the range in inter-BS information exchange regarding local system throughput. Pico-BS $n$ shares the system throughput values among neighboring BSs within a range of distances, $R$. The set of BSs that inform its observed system throughput to pico-BS $n$ is denoted as $\mathcal{N}_{\mathrm{BS}}^{(n)}$. The system throughput at time $t$ that is obtained at pico-BS $n$ using inter-BS information exchange, $\bar{C}_{n}[t]$, is represented as

$$
\begin{aligned}
\bar{C}_{n}[t] & =\left(\prod_{m \in \mathcal{N}_{\mathrm{BS}}^{(n)} \cup\{n\}} \prod_{k \in \mathcal{K}_{m}[t]} c_{k}[t]\right)^{1 / \sum_{m \in \mathcal{N}_{\mathrm{BS}}^{(n)} \cup[n]}\left|\mathcal{K}_{m}[t]\right|} \\
& =\left(\prod_{m \in \mathcal{N}_{\mathrm{BS}}^{(n)} \cup\{n\}} C_{m}[t]^{\left|\mathcal{K}_{m}[t]\right|}\right)^{1 / \sum_{m \in N_{\mathrm{BS}}^{(n)} \cup[n]}\left|\mathcal{K}_{m}[t]\right|} .
\end{aligned}
$$

To calculate $\bar{C}_{n}[t]$, each BS $m$ shares $C_{m}[t]$ and $\left|\mathcal{K}_{m}[t]\right|$ levels (only two scalar numbers) with neighboring BSs through the backhaul at each time $t$ using inter-BS information exchange. Assuming a uniform distribution of BSs, the re- 
quired amount of inter-BS exchange information is inversely proportional to $R^{2}$. The conventional method employing the LRIE uses $\bar{C}_{n}[t]$ instead of $\bar{C}[t]$ in the control of $q_{n}[t]$ at pico-BS $n$. Please refer to [11] to view more detailed discussions and quantitative evaluation results.

\subsection{ASSC}

As described above, in the conventional method in [10], the step size in updating activation probability $q_{n}[t]$ is fixed at $\pm \varepsilon$ regardless of the time variation in system throughput level $\bar{C}[t]$ or $\bar{C}_{n}[t]$ when the LRIE is applied. However, it is considered that both the convergence rate and the stability after convergence of the iterative algorithm in the conventional method can be improved by updating $q_{n}[t]$ with a larger step size when the absolute value of $\Delta \bar{C}[t]$ or $\Delta \bar{C}_{n}[t]$ is large, and updating $q_{n}[t]$ with a smaller step size when the absolute value of $\Delta \bar{C}[t]$ or $\Delta \bar{C}_{n}[t]$ is small. Therefore, the ASSC in [12] for the conventional method uses the following formula instead of (6) for updating $q_{n}[t]$.

$$
q_{n}[t+1]=q_{n}[t]+\varepsilon \cdot \Delta \bar{C}[t] \cdot\left(s_{n}[t]-s_{n}[t-1]\right)
$$

When the LRIE is applied, $\Delta \bar{C}[t]$ is replaced by $\Delta \bar{C}_{n}[t]$ in (8). In (8), the effective step size in updating $q_{n}[t]$ is $\varepsilon \cdot \Delta \bar{C}[t]$. The ASSC aims at improving both the convergence rate of the iterative algorithm and increasing the system throughput after convergence by adaptively changing the step size in updating the activation probability depending on the time variation in the system throughput levels.

\section{Proposed Method}

In the proposed method, the overall system bandwidth is divided into multiple frequency blocks; thus, $|\mathcal{F}|$ is set to greater than one. The proposed method independently performs activation control on a per frequency block basis at each BS. By performing the frequency block-dependent activation control, we can increase the granularity of the activation/deactivation control and achieve an ICIC effect in addition to the overall reduction in inter-cell interference.

A straightforward way to perform frequency blockdependent activation control is to apply the conventional method described in Sect. 3.1 to each frequency block independently. However, the higher granularity in the activation/deactivation control may result in a longer convergence time. To address this problem, we propose a method in which the number of activated frequency blocks is controlled in a probabilistic manner in addition to the control in activation probability of each frequency block.

The proposed method performs activation control at each pico-BS $n$ based on $\bar{C}[t]$ or $\bar{C}_{n}[t]$ similar to the conventional method. In the following description, assuming that the proposed method is conducted with the LRIE, $\bar{C}_{n}[t]$ is used for the proposed activation control. We note that the required amount of inter-BS exchange information in the proposed method is not dependent on the number of frequency blocks, since the proposed method shares only the system throughput levels per BS among neighboring BSs via the inter-BS information exchange, which is the same as in the method in [10], and the amount of inter-BS exchange information is not a function of the number of frequency blocks. The proposed method is a decentralized approach and we describe below the control at a certain pico-BS $n$ of interest.

The proposed method defines parameter $b_{n, m}[t]$, which represents the probability that the number of activated frequency blocks of pico-BS $n$ is $m$ at time $t$. The proposed method also defines parameter $q_{n, f}[t]$, which is the relative probability that frequency block $f$ of pico-BS $n$ is activated at time $t$. The proposed method independently updates $b_{n, m}[t]$ and $q_{n, f}[t]$ at each pico-BS $n$ based on the time variation of local system throughput $\bar{C}_{n}[t]$ and activation states of pico-BS $n$ at each frequency block $f$ during the same time instances.

The activation/deactivation state of frequency block $f$ in pico-BS $n$ at time $t$ is represented by $s_{n, f}[t] \in\{1,0\}$. The number of frequency blocks of pico-BS $n$ that are activated at time $t$ is represented as $m_{n}[t]$. In the proposed method, the time range of the system observations used for activation/deactivation control is assumed to be $T$ time slots.

At time $t, \tilde{C}_{n, m}[t]$, which is the linear-weighted average of the system throughput when $m$ frequency blocks of picoBS $n$ are activated over the past $T$ discrete time, is calculated at pico-BS $n$. Term $\tilde{C}_{n, m}[t]$ is represented as

$$
\tilde{C}_{n, m}[t]=\frac{\sum_{i=t-T+1, m_{n}[i]=m}^{i=t}(T-t+i) \bar{C}_{n}[i]}{\sum_{i=t-T+1, m_{n}[i]=m}^{i=t}(T-t+i)} .
$$

The proposed method updates $b_{n, m}[t]$ according to the $\left\{\tilde{C}_{n, m}[t]\right\}$ values for all $m$ using the following equation.

$$
\begin{aligned}
& b_{n, m}[t+1]= \begin{cases}b_{n, m}[t]+\varepsilon_{b}[t], & m=m_{\max }[t] \\
b_{n, m}[t]+\varepsilon_{b}[t] / \beta, & m=m_{\max }[t] \pm 1 \\
b_{n, m}[t]-\varepsilon_{b}[t], & m=m_{\min }[t] \\
b_{n, m}[t]-\varepsilon_{b}[t] / \beta, & m=m_{\min }[t] \pm 1 \\
b_{n, m}[t], & \text { Otherwise }\end{cases} \\
& m_{\max }[t]=\arg \max _{m} \tilde{C}_{n, m}[t], m_{\min }[t]=\arg \min _{m} \tilde{C}_{n, m}[t] .
\end{aligned}
$$

Here, control step size $\varepsilon_{b}[t]$ is represented as

$$
\varepsilon_{b}[t]=\frac{\tilde{C}_{n, m_{\max }[t]}[t]-\tilde{C}_{n, m_{\min }[t]}[t]}{\left(\tilde{C}_{n, m_{\max }[t]}[t]+\tilde{C}_{n, m_{\min }[t]}[t]\right) / 2} \varepsilon_{1} .
$$

Term $\varepsilon_{1}$ is a small positive constant that corresponds to the unit step size. We note that the minimum and maximum values of $b_{n, m}[t]$ are limited to $\rho$ and $1-\rho$, respectively, where $\rho$ is a small positive constant to avoid $b_{n, m}[t]$ being fixed to 0 or 1 . Term $\beta$ is a constant greater than 1 . Thus, by increasing $b_{n, m}[t]$ with $m$, which obtains the highest system throughput in the past $T$ time, and decreasing $b_{n, m}[t]$ with $m$, which obtains the minimum system throughput, we can expect that $m_{n}[t+1]$ becomes a more appropriate value 
from the viewpoint of the increase in the system throughput. The reason why $b_{n, m}[t]$ in the vicinity of the best or worst $m$ is also updated with the smaller step size of $\varepsilon_{b}[t] / \beta$ is to improve the convergence rate of the iterative algorithm. Control step size $\varepsilon_{b}[t]$ in (11) includes the ASSC concept. That is, we aim at improving the convergence rate of the iterative algorithm and increasing the system throughput after convergence by adaptively changing the step size when updating the activation probability depending on the time variation in the system throughput levels.

Pico-BS $n$ also calculates $\tilde{C}_{n, f}^{\mathrm{ON}}[t]$ and $\tilde{C}_{n, f}^{\mathrm{OFF}}[t]$, which are the average system throughput levels when frequency block $f$ of pico-BS $n$ is activated or deactivated, respectively, during the past $T$ discrete time. $\tilde{C}_{n, f}^{\mathrm{ON}}[t]$ and $\tilde{C}_{n, f}^{\mathrm{OFF}}[t]$ are given as

$$
\begin{aligned}
\tilde{C}_{n, f}^{\mathrm{ON}}[t] & =\frac{\sum_{i=t-T+1}^{i=t}(T-t+i) s_{n, f}[i] \bar{C}_{n}[i]}{\sum_{i=t-T+1}^{i=t}(T-t+i) s_{n, f}[i]} \\
\tilde{C}_{n, f}^{\mathrm{OFF}}[t] & =\frac{\sum_{i=t-T+1}^{i=t}(T-t+i)\left(1-s_{n, f}[i]\right) \bar{C}_{n}[i]}{\sum_{i=t-T+1}^{i=t}(T-t+i)\left(1-s_{n, f}[i]\right)}
\end{aligned} .
$$

The proposed method updates $q_{n, f}[t]$ based on $\tilde{C}_{n, f}^{\mathrm{ON}}[t]$ and $\tilde{C}_{n, f}^{\mathrm{OFF}}[t]$ using the following equation.

$$
q_{n, f}[t+1]=q_{n, f}[t]+\frac{\tilde{C}_{n, f}^{\mathrm{ON}}[t]-\tilde{C}_{n, f}^{\mathrm{OFF}}[t]}{\left(\tilde{C}_{n, f}^{\mathrm{ON}}[t]+\tilde{C}_{n, f}^{\mathrm{OFF}}[t]\right) / 2} \varepsilon_{2} .
$$

Term $\varepsilon_{2}$ is a small positive constant that corresponds to the unit step size. We note that the minimum and maximum values of $q_{n, f}[t]$ are limited to $\rho$ and $1-\rho$, respectively. Thus, $q_{n, f}[t]$ of the frequency block with which the system throughput has increased when activated over the past $T$ time is increased; otherwise, it is decreased. The updating of $q_{n, f}[t]$ using (13) also utilizes the ASSC concept.

At the next time, $t+1$, the number of frequency blocks of pico-BS $n$ to be activated, $m_{n}[t+1]$, is determined in a probabilistic manner based on the updated $\left\{b_{n, m}[t+1]\right\}$ for all $m$. Next, $m_{n}[t+1]$ frequency blocks to be activated are determined based on $\left\{q_{n, f}[t+1]\right\}$ for all $f$ in a probabilistic manner. BS association of all users is updated based on the determined activation/deactivation states of all the BSs. By repeating this process over time, the activation/deactivation control is actualized for all frequency blocks of all the BSs to achieve the ICIC effect and traffic load balancing adapted to the BS and user distribution.

Regarding throughput averaging interval $T$ in the proposed method, we can expect that a longer $T$ is beneficial for a faster convergence rate. This is because the undesirable impact of the transition of activation/deactivation states of other pico-BSs on the control of the activation probability is mitigated by the averaging of the system throughput observed over the longer past time duration. However, in a realistic dynamic user mobility environment, the use of an excessively long $T$ may result in a reduction in system throughput due to the degraded tracking ability of the proposed activation control against the user mobility.

\section{Numerical Results}

\subsection{Simulation Assumptions}

Table 1 gives the simulation parameters. The system bandwidth of all BSs is set to $w_{\text {tot }}=9 \mathrm{MHz}$. We basically assume that different frequency bands are allocated to macro- and pico-BSs. However, as shown later in Fig. 7, the case where the same frequency band is shared by macro- and pico-BSs is also tested. The macro-BSs, pico-BSs, and user terminals are placed at random locations within a wrap-around $5 \times 5$ square kilometer system coverage area based on the Poisson point process (PPP). The node densities of the macro-BSs, pico-BSs, and user terminals are set to $1, D_{\text {pico }}$, and 30 per square kilometer, respectively. Density $D_{\text {pico }}$ is parameterized in the following evaluations. The transmission power levels of the macro- and pico-BSs are $46 \mathrm{dBm}$ and $30 \mathrm{dBm}$, respectively. OFDMA is assumed as the multiple access scheme. Omni-directional antennas with the antenna gain of $14 \mathrm{dBi}$ are assumed for both macro- and pico-BSs for simplicity. As the propagation model, distance-dependent path loss and lognormally distributed random shadowing are assumed with the parameters given in Table 1. The receiver noise power density of the user terminals including the noise figure is set to $-165 \mathrm{dBm} / \mathrm{Hz}$. The CRE method [17] is used for user association. The user throughput is calculated based on the Shannon formula. Note that the transmission power of the BS, which does not temporally serve any user terminal as a result of the user association process, is assumed to be lowered to the $10 \%$ level of its maximum transmission power since the data channel is not transmitted ( $10 \%$ is for transmitting reference signals, the broadcast channel, etc.). As the system throughput, we use the geometric mean user throughput.

In the proposed method, the number of frequency blocks, $F=|\mathcal{F}|$, is parameterized from 1 to 5 . The range in inter-BS information exchange, $R$, is set to $1.3 \mathrm{~km}$ and

\begin{tabular}{|c|c|c|}
\hline \multicolumn{2}{|c|}{ System bandwidth, $w_{\text {tot }}$} & $9 \mathrm{MHz}$ \\
\hline \multicolumn{2}{|c|}{ Number of frequency blocks, $\mid \mathcal{F}$} & $1 \sim 5$ \\
\hline \multicolumn{2}{|c|}{ Averaging interval, $T$} & $2 \sim 20$ \\
\hline \multirow{3}{*}{ Node density } & Macro-BS & $1 / \mathrm{km}^{2}$ \\
\hline & Pico-BS & $D_{\text {pico }} / \mathrm{km}^{2}$ \\
\hline & User terminal & $30 / \mathrm{km}^{2}$ \\
\hline \multirow{2}{*}{$\begin{array}{l}\text { BS transmission } \\
\text { power }\end{array}$} & Macro-BS & $46 \mathrm{dBm}$ \\
\hline & Pico-BS & $30 \mathrm{dBm}$ \\
\hline \multicolumn{2}{|c|}{ Distance-dependent path loss } & $128.1+37.6 \log _{10}(r), r:$ kilometers \\
\hline \multicolumn{2}{|c|}{ Shadowing } & $\begin{array}{l}\text { Lognormal shadowing with standard } \\
\text { deviation of } 8 \mathrm{~dB} \text { and inter-site correlation of } 0.5\end{array}$ \\
\hline \multicolumn{2}{|c|}{$\begin{array}{l}\text { Receiver noise power density } \\
\text { (including noise figure) }\end{array}$} & $-165 \mathrm{dBm} / \mathrm{Hz}$ \\
\hline \multicolumn{2}{|c|}{ User association } & CRE method \\
\hline
\end{tabular}
$1.0 \mathrm{~km}$ for static and dynamic user mobility environments,

Table 1 Simulation parameters. 
respectively. Parameters $\varepsilon_{1}$ and $\varepsilon_{2}$ are set to 0.3 and 1.0 , respectively, except for Fig. 7 where both $\varepsilon_{1}$ and $\varepsilon_{2}$ are set to 1.0. Term $\beta$ is set to $|\mathcal{F}|$. Parameter $\rho$ is set to 0.01 . These parameter settings are experimentally determined based on a pre-performed computer simulation so that the system throughput is increased the most. In the following evaluation, in addition to the proposed method with the LRIE and ASSC, the conventional method and proposed method without the LRIE and ASSC are evaluated for comparison.

\subsection{Simulation Results}

First, we show the operational tendency of the activation control for each frequency block in the proposed method. Figure 1 shows an example of the geometrical distribution of each node where a $1 \times 1$-square kilometer area from the entire $5 \times 5$-square kilometer system coverage is shown for visibility. Figure 2 shows the controlled $q_{n, f}$ values of the proposed method after convergence in each frequency block of each BS. We assume that the number of frequency blocks, $F$, is three in this evaluation. The node densities of the macro-BSs, pico-BSs, and user terminals are set to 0,5 , and 20 per square kilometer, respectively, only in this evaluation for the sake of clarity of the figure. Figures 1 and 2 show that since relatively many user terminals are distributed in the upper right region, $q_{n, f}$ of the pico-BS in that region, e.g., pico-BS 6 , is controlled to be a relatively high value.

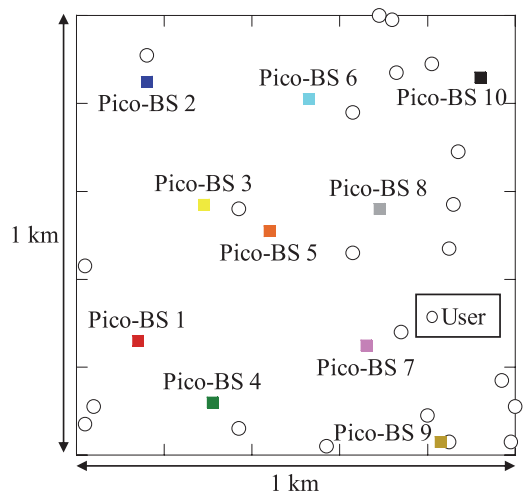

Fig. 1 Example of node arrangement.

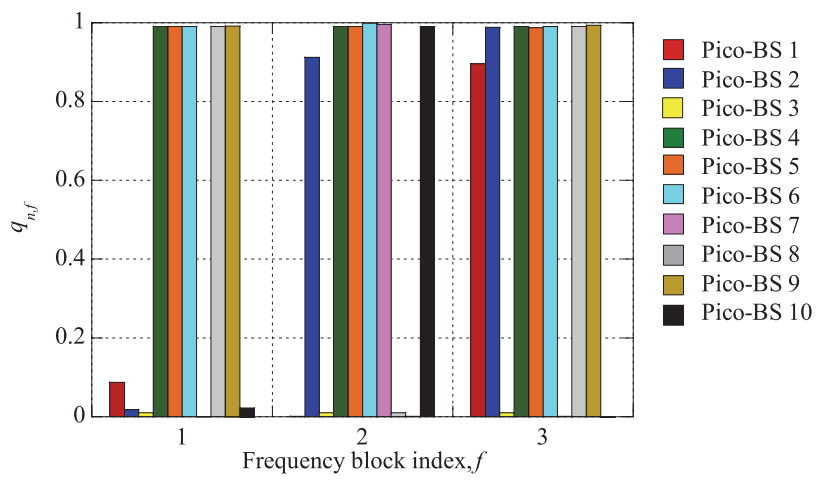

Fig. 2 Probability $q_{n, f}$ of each BS in Fig. 1.
The $q_{n, f}$ value of the pico-BS located in the region where the number of user terminals is relatively low, e.g., pico-BS 3 , is controlled to be low. Furthermore, there is a tendency that neighboring BSs tend to have high $q_{n, f}$ values at different frequency blocks, and that BSs that are distant from each other tend to have high $q_{n, f}$ values in the same frequency block. So it is understood that the proposed method autonomously obtains the ICIC effect.

Figure 3 shows the system throughput as a function of the number of iterations in the iterative algorithm of the activation/deactivation control. Density $D_{\text {pico }}$ is 20 . In the proposed method, $F$ and $T$ are set to 3 and 10, respectively. The proposed and conventional activation controls achieve higher system throughput than the case without activation control. The proposed method achieves greater system throughput than the conventional method. This is because the granularity in the activation/deactivation control and ICIC effect for a given distribution of BSs and user terminals is increased. The convergence rate and stability after convergence of the proposed method are enhanced using the LRIE and ASSC. This is because the LRIE reduces the undesirable control in updating the activation probability of a particular BS based on the system throughput observed far away from that BS, which is effectively not related to the activation/deactivation state of that BS. The ASSC further improves the convergence rate and stability after convergence.

Figure 4 shows the system throughput after convergence of the proposed method as a function of the number of frequency blocks, $F$. As $F$ increases, the system throughput of the proposed method is increased. This is due to the increased granularity in the activation/deactivation control and ICIC effect for a given distribution of BSs and user terminals. Additional throughput gain by further increasing the number of frequency blocks, $F$, beyond four is marginal.

We note that in the simulation evaluation, instantaneous fading is not considered for simplicity; therefore, the frequency selectivity of each user channel that occurs in the multipath fading channel is not considered. However, in the proposed method, the influence of the frequency selectivity of each user channel on the activation/deactivation of the respective frequency blocks and the resulting number of

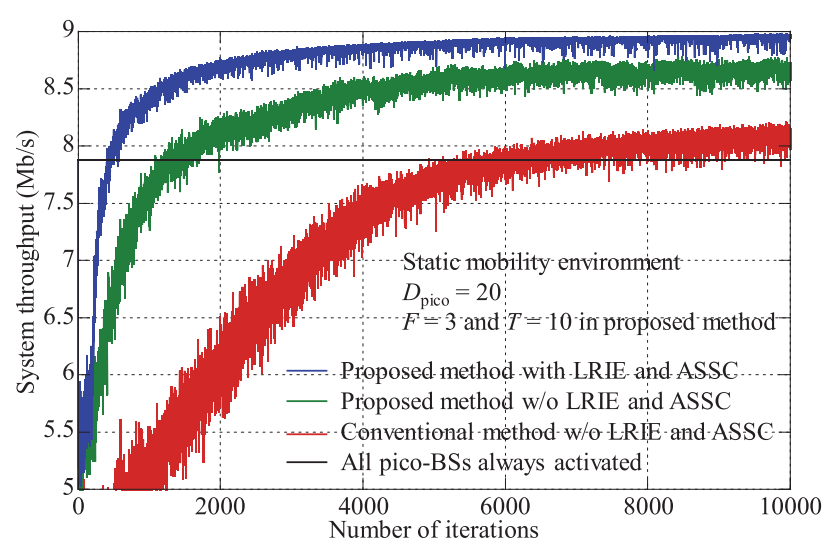

Fig. 3 System throughput as a function of number of iterations. 


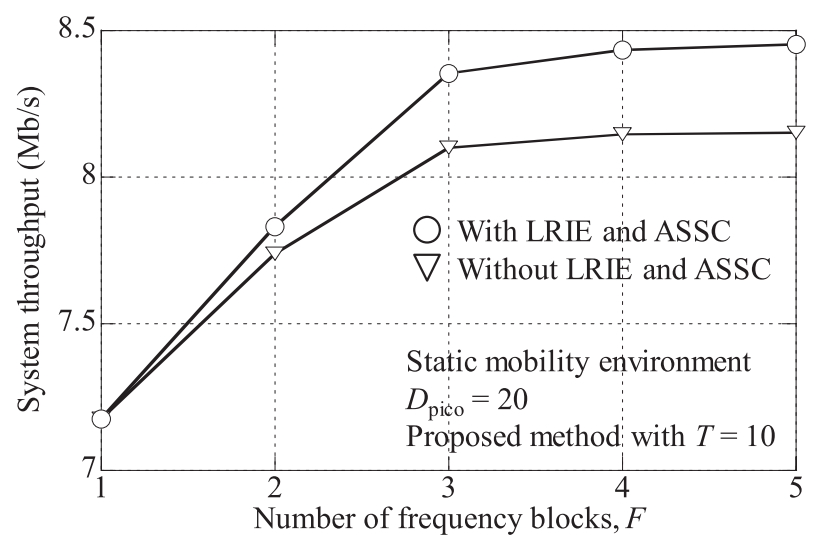

Fig. 4 System throughput as a function of number of frequency blocks.

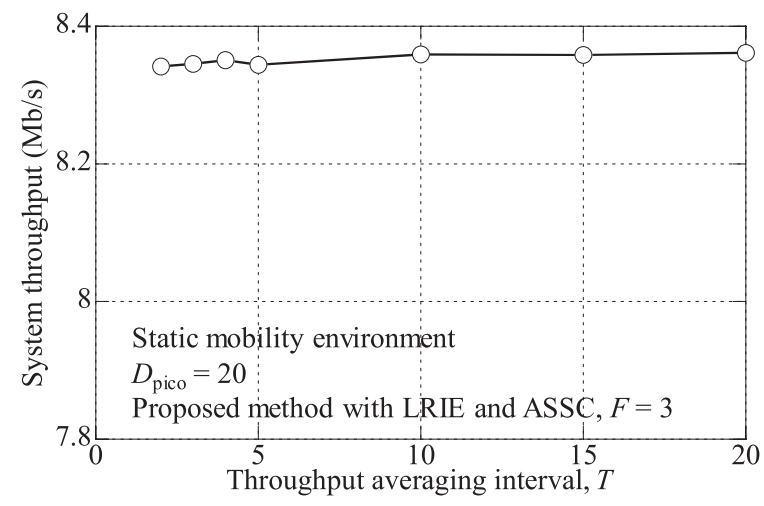

Fig. 5 System throughput after convergence as a function of $T$.

activated frequency blocks are considered not to be significant. This is because the update time interval of the activation control in the proposed method is generally longer than the frequency-selective fading period. Each user channel of each frequency block averaged over the update time interval of the activation control tends to be frequency nonselective which simply depends on the average path loss such as distance-dependent loss and random shadowing. In the following evaluation, we set $F$ to three.

Next, we verify the improvement in the convergence property of the proposed method using the system throughput observations and corresponding activation/deactivation states for a wider range of past time windows, $T$, than the two time slots in a static user mobility environment.

Figure 5 shows the system throughput after convergence as a function of $T$ in the proposed method. From the figure, we confirm that the dependence of the system throughput after convergence on $T$ is not noticeable. Figure 6 shows the number of iterations required for convergence as a function of $T$ in the proposed method. The convergence condition is defined as the number of iterations that first reaches the $95 \%$ value of the system throughput after a sufficient number of iterations. We see that a longer $T$ is beneficial for a faster convergence rate as expected. However, in a realistic dynamic user mobility environment where the user terminal moves over time, the use of an excessively

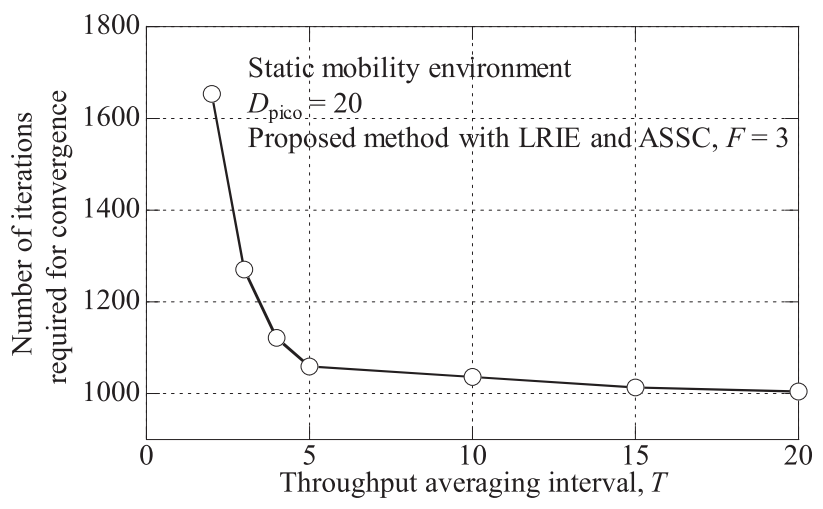

Fig. 6 Number of repetitions required for convergence as a function of $T$.

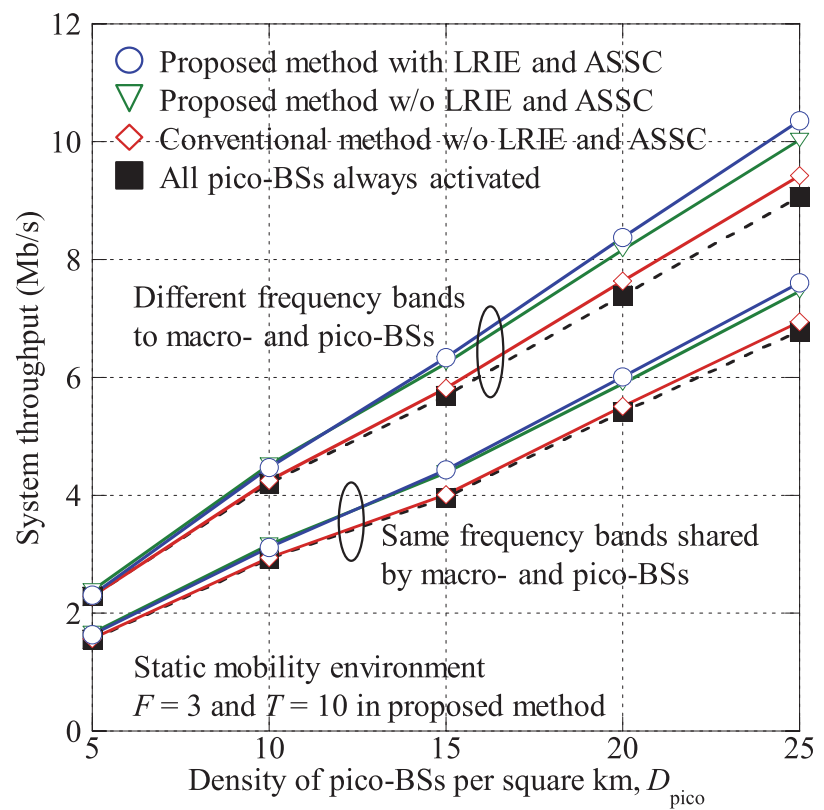

Fig. 7 System throughput as a function of pico-BS density.

long $T$ may result in a reduction in system throughput due to the degraded tracking ability of the proposed activation control against the user mobility (change in the distribution of user terminals). Therefore, we evaluate the proposed method in a dynamic user mobility environment later.

Figure 7 shows the system throughput as a function

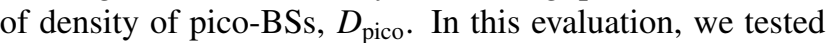
two frequency reuse scenarios: (1) different frequency bands are allocated to macro-BSs and pico-BSs, and (2) the same frequency band is shared by macro- and pico-BSs. Figure 7 reveals that the effect of the proposed method is increased as $D_{\text {pico }}$ is set high. For example, when $D_{\text {pico }}$ is increased from 5 to 25 , an additional system throughput gain of approximately $10 \%$ is obtained using the proposed method. This is because in this case, the inter-cell interference caused by the pico-BS is a dominant degradation factor in the system throughput and the proposed method adaptively activates/deactivates the respective frequency blocks 
of each BS achieving the ICIC effect in addition to an overall reduction in the inter-cell interference. Furthermore, the performance gain using the proposed method is increased when different frequency bands are allocated to macro- and pico-BSs. This is because in this scenario, the users connected to the pico-BSs are free from the interference from the high-transmission power macro-BSs and the inter-cell interference caused by the pico-BS significantly affects the achievable throughput levels. We note that this is actually the scenario expected in the 5th generation mobile communication system where many pico-BSs are deployed and a new millimeter-wave frequency band is allocated to the set of pico-BSs. Thus, the proposed method is effective in utilizing the pico-BSs and new frequency band in the 5th generation mobile communication system.

Hereafter, we investigate the performance of the proposed method in a dynamic user mobility environment. The random waypoint (RWP) model [20] is used to simulate the user mobility. The velocity of user $k$ is denoted as $v_{k}$. In the following evaluation, $v_{k}$ of each user $k$ is randomly given based on a uniform distribution within the range of $0 \mathrm{~km} / \mathrm{h}$ to $4 \mathrm{~km} / \mathrm{h}$ to simulate a pedestrian environment. User $k$ moves at $v_{k}$ toward the destination, which is randomly selected within the system coverage for each user. After arriving at the destination, user $k$ takes a break with thinking time. Thinking time is set to $10 \mathrm{~s}$. After the break, user $k$ randomly determines a new destination and $v_{k}$, and restarts movement.

Figure 8 shows the time variation of the system throughput in the RWP-based dynamic user mobility environment. Density $D_{\text {pico }}$ is set to 20 . In the proposed method, $F$ and $T$ are set to 3 and 5, respectively. In the proposed and conventional activation control methods, the update time interval of the activation control is set to $10 \mathrm{~ms}$. The proposed method achieves greater system throughput even in dynamic user mobility environments compared to the conventional two cases. Thus, the activation control of the proposed method successfully tracks the dynamic changes in the user distribution due to mobility. The application of the LRIE and ASSC to the proposed method improves the tracking ability of the activation control to the dynamic change in user distribution, which results in increased achievable throughput.

Figure 9 shows the cumulative distribution of the system throughput in the RWP-based user mobility environment. Figure 9 is obtained by statistically processing the throughput level samples, which are shown in Fig. 8, over $500 \mathrm{~s}$. Therefore, the performance shown in Fig. 9 includes both the convergence characteristics from the initial time and that after the convergence, which is predominantly determined by the tracking ability of the activation control against the change in user locations over time due to mobility. In addition, for the proposed method, $T$ is set as a parameter in the range of 2 to 20 . The update time interval of the activation/deactivation control is set to $10 \mathrm{~ms}$. The case where all the BSs are always activated is also tested. In Fig. 9, The probability on the y-axis means the outage

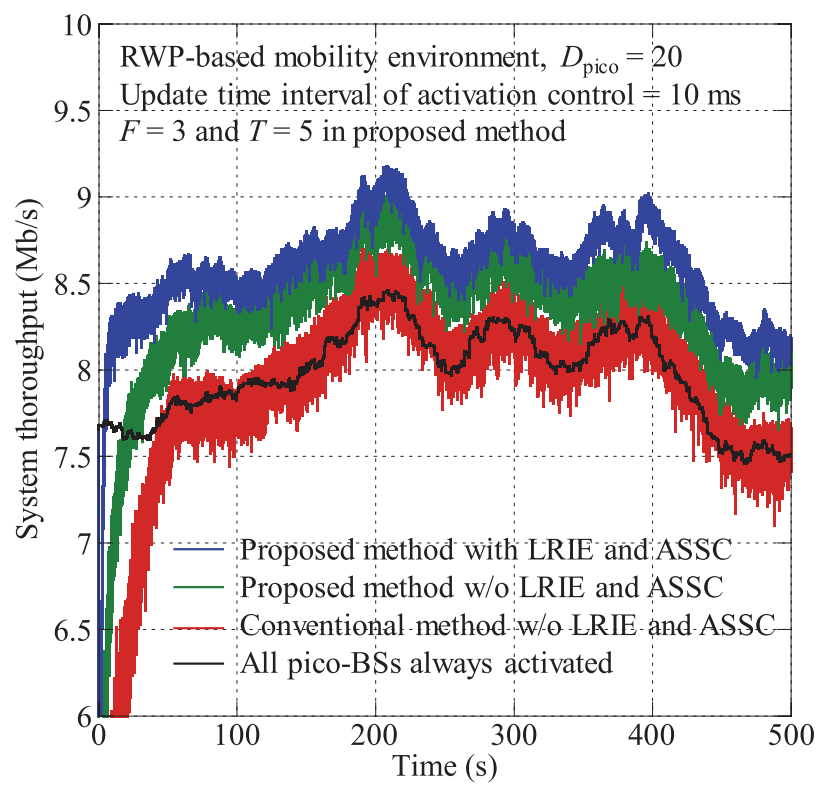

Fig. 8 Time variation of system throughput in RWP-based dynamic user mobility environment.

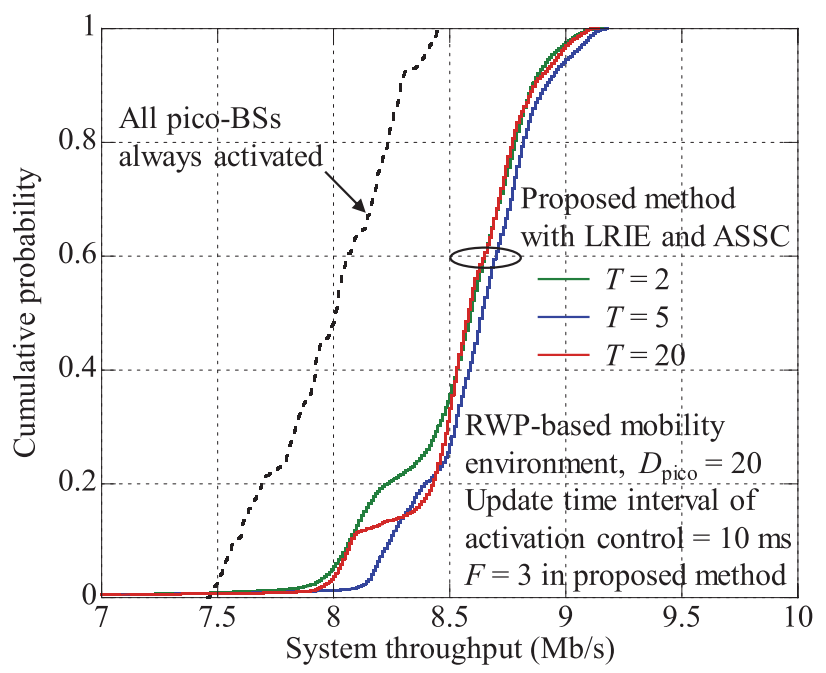

Fig. 9 Cumulative distribution of system throughput in RWP-based user mobility environment.

probability for serving the system throughput levels on the $\mathrm{x}$-axis. The figure shows that the outage probability of the system throughput achieved by the proposed method is significantly better compared to the case without BS activation control thanks to the decreased inter-cell interference and obtained ICIC effect. In the proposed method, when $T$ is relatively short such as two, the advantage of the accuracy in the control of the activation/deactivation by averaging the past system observations is limited. On the other hand, when $T$ is increased to 20 , the tracking ability of the proposed activation control against the user mobility is degraded. As a result, the outage probability of the system throughput is the best when $T$ is set to 5 in the range evaluated, except for system throughput levels between approximately 8.25 to 
$8.40 \mathrm{Mb} / \mathrm{s}$ where $T$ of 20 achieves the lowest outage probability. Thus, it is important to decide the value of $T$ considering the moving speed of the user terminal.

\section{Conclusion}

In this paper, we proposed a novel decentralized frequency block-dependent online probabilistic activation control of BSs in heterogeneous networks. By defining multiple frequency blocks in an overall system bandwidth and performing activation control on a per frequency block basis, the proposed method achieves the ICIC effect and traffic load balancing in addition to an overall reduction in the inter-cell interference by appropriately deactivating BSs for a given BS and user distribution. The use of the LRIE and ASSC significantly improves the convergence performance of the iterative algorithm in the proposed method. The evaluation conducted in a dynamic user mobility environment reveals the tracking ability of the proposed method regarding the dynamic changes in the user distribution due to mobility. The proposed method is especially effective when the density of pico-BSs is high and different frequency bands are allocated between macro- and pico-BSs. This is actually the scenario expected in the 5th generation mobile communication system where many pico-BSs are deployed and a new millimeter-wave frequency band is allocated to the set of pico-BSs. Thus, the proposed method can be a promising component technique in order to utilize effectively the picoBSs and new frequency band in the 5th generation mobile communication system.

\section{References}

[1] ITU-R, "IMT Vision - Framework and overall objectives of the future development of IMT for 2020 and beyond," Recommendation M.2083-0, Sept. 2015.

[2] 3GPP TR38.913 (V0.4.0), "Study on scenarios and requirements for next generation access technologies (Release 14)," June 2016.

[3] 3GPP TR38.912 (V0.0.1), "Study on new radio access technology (Release 14)," June 2016.

[4] A. Damnjanovic, J. Montojo, Y. Wei, T. Ji, T. Luo, M. Vajapeyam, T. Yoo, O. Song, and D. Malladi, "A survey on 3GPP heterogeneous networks," IEEE Wireless Commun., vol.18, no.3, pp.10-21, June 2011.

[5] A. Ghosh, N. Mangalvedhe, R. Ratasuk, B. Mondal, M. Cudak, E. Visotsky, T.A. Thomas, J.G. Andrews, P. Xia, H.S. Jo, H.S. Dhillon, and T.D. Novlan, "Heterogeneous cellular networks: From theory to practice," IEEE Commun. Mag., vol.50, no.6, pp.54-64, June 2012.

[6] S. Zhou, J. Gong, Z. Yang, S. Zhou, Z. Niu, and P. Yang, "Green mobile access network with dynamic base station energy saving," Proc. ACM MobiCom'09, pp.1-3, Beijing, China, Sept. 2009.

[7] Y.S. Soh, T.Q.S. Quek, M. Kountouris, and H. Shin, "Energy efficient heterogeneous cellular networks," IEEE J. Sel. Areas Commun., vol.31, no.5, pp.840-850, May 2013.

[8] S. Zhang, J. Wu, J. Gong, S. Zhou, and Z. Niu, "Energy-optimal probabilistic base station sleeping under a separation network architecture," Proc. IEEE GLOBECOM2014, pp.4239-4244, Austin, U.S.A., Dec. 2014.

[9] Y. Yamazaki and K. Higuchi, "Online probabilistic activation control of picocells for system throughput maximization in heterogeneous networks," Proc. IEEE ISPACS 2015, Bali, Indonesia, Nov. 2015.
[10] A. Ujiie and K. Higuchi, "Improved methods for online probabilistic activation control of base stations based on observed system throughput in heterogeneous networks," Proc. IEEE APWCS2016, Tokyo, Japan, Aug. 2016.

[11] K. Takahashi and K. Higuchi, "On the range in inter-base station information exchange in online probabilistic activation control of base stations based on observed system throughput," Proc. IEEE APWCS2016, Tokyo, Japan, Aug. 2016.

[12] R. Ochiai, Y. Kishiyama, and K. Higuchi, "Adaptive step size control for update of activation probability in online probabilistic BS activation control method," Proc. WPMC2018, Chiang Rai, Thailand, Nov. 2018.

[13] G. Boudreau, J. Panicker, N. Guo, R. Chang, N. Wang, and S. Vrzic, "Interference coordination and cancellation for $4 \mathrm{G}$ networks," IEEE Commun. Mag., vol.47, no.4, pp.74-81, April 2009.

[14] T.D. Novlan, R.K. Ganti, A. Ghosh, and J.G. Andrews, "Analytical evaluation of fractional frequency reuse for OFDMA cellular downlink," IEEE Trans. Wireless Commun., vol.10, no.12, pp.4294 4305, Dec. 2011

[15] K. Shimada, Y. Kishiyama, and K. Higuchi, "Frequency blockdependent online probabilistic activation control of base stations in heterogeneous networks," Proc. WPMC2018, Chiang Rai, Thailand, Nov. 2018.

[16] F. Ishikawa, Y. Kishiyama, and K. Higuchi, "Decentralized probabilistic frequency-block activation control for inter-cell interference coordination and traffic load balancing," Proc. IEEE VTC2019-Fall, Honolulu, U.S.A., Sept. 2019.

[17] R. Madan, J. Borran, A. Sampath, N. Bhushan, A. Khandekar, and T. $\mathrm{Ji}$, "Cell association and interference coordination in LTE-A cellular networks,” IEEE J. Sel. Areas Commun., vol.28, no.9, pp.1479_ 1489, Dec. 2010

[18] F. Kelly, "Charging and rate control for elastic traffic," Eur. Trans. Telecommun., vol.8, no.1, pp.33-37, 1997.

[19] H. Seki, T. Kobayashi, and D. Kimura, "Selection of component carriers using centralized baseband pooling for LTE-advanced heterogeneous networks," IEICE Trans. Commun., vol.E96-B, no.6, pp.1288-1296, June 2013.

[20] T. Camp, J. Boleng, and V. Davies, "A survey of mobility models for ad hoc network research," Wirel. Commun. Mob. Comput., vol.2, no.5, pp.483-502, 2002. 10.1002/wcm.72.

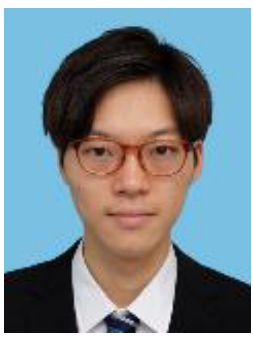

Fumiya Ishikawa received the B.E. degree from Tokyo University of Science, Noda, Japan in 2019. He is currently working towards his M.E. degree in the Department of Electrical Engineering, Tokyo University of Science, Noda, Japan. His research interest includes wireless communications. He is a student member of the IEICE and IEEE. 


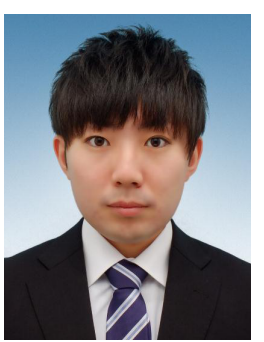

Keiki Shimada received the B.E. and M.E. degrees from Tokyo University of Science, Noda, Japan in 2018 and 2020, respectively. In 2020, he joined NTT DOCOMO, INC.

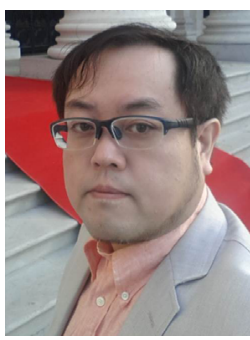

Yoshihisa Kishiyama is a Manager of $5 \mathrm{G}$ Laboratories in NTT DOCOMO, INC. He received his B.E., M.E., and Ph.D. degrees from Hokkaido University, Sapporo, Japan in 1998, 2000, and 2010, respectively. Since he joined NTT DOCOMO in 2000, he has been involved in research and development on $4 \mathrm{G} / 5 \mathrm{G}$ radio access technologies. In 2012, he received the International Telecommunication Union Association of Japan (ITU-AJ) Award for contributions to LTE standardization.

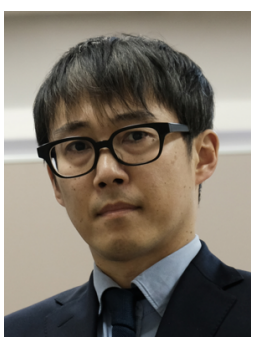

Kenichi Higuchi received the B.E. degree from Waseda University, Tokyo, Japan, in 1994, and received the Dr.Eng. degree from Tohoku University, Sendai, Japan in 2002. In 1994, he joined NTT Mobile Communications Network, Inc. (now, NTT DOCOMO, INC.). While with NTT DOCOMO, INC., he was engaged in the research and standardization of wireless access technologies for wideband DS-CDMA mobile radio, HSPA, LTE, and broadband wireless packet access technologies for systems beyond IMT-2000. In 2007, he joined the faculty of the Tokyo University of Science and currently holds the position of Professor. His current research interests are in the areas of wireless technologies and mobile communication systems, including advanced multiple access, radio resource allocation, inter-cell interference coordination, multiple-antenna transmission techniques, signal processing such as interference cancellation and turbo equalization, and issues related to heterogeneous networks using small cells. He was a co-recipient of the Best Paper Award of the International Symposium on Wireless Personal Multimedia Communications in 2004 and 2007, a recipient of the Young Researcher's Award from the IEICE in 2003, the 5th YRP Award in 2007, the Prime Minister Invention Prize in 2010, and the Invention Prize of Commissioner of the Japan Patent Office in 2015. He is a member of the IEEE. 